Notfall Rettungsmed 2015 · 18:5-6

DOI 10.1007/s10049-014-1978-9

Online publiziert: 23. Januar 2015

(c) Springer-Verlag Berlin Heidelberg 2015
B.W. Böttiger ${ }^{1} \cdot$ C. Waydhas ${ }^{2} \cdot$ C. Wrede ${ }^{3}$

${ }^{1}$ Klinik für Anästhesiologie und Operative Intensivmedizin, Universitätsklinikum Köln, Köln

${ }^{2}$ Klinik und Poliklinik für Unfallchirurgie, Universitätsklinikum Essen, Essen

${ }^{3}$ Notfallzentrum mit Rettungsstelle, HELIOS Klinikum Berlin-Buch, Berlin

\title{
Notfall- und Rettungsmedizin - offen für Neues
}

Liebe Leserinnen und Leser,

liebe Autorinnen und Autoren,

vor 7 Jahren gab es für die Notfall + Rettungsmedizin den ersten wichtigen „wissenschaftlichen Ritterschlag". Notfall + Rettungsmedizin wurde im Institute of Scientific Information (ISI) in den Science Citation Report aufgenommen. 2009 war das erste Jahr mit Impact Factor. Auch wenn die Rolle des Impact Factor für wissenschaftliche Zeitschriften zunehmend kritisch gesehen wird [1], so ist er derzeit für eine wissenschaftliche Zeitschrift noch unverzichtbar.

Der zweite große Schritt für unsere Zeitschrift soll die Listung in PubMed, der online Bibliothek der US National Library - National Institutes (NLM-NIH) sein.

Die Aufnahme unserer Inhalte

in das von der US-Regierung

finanzierte System würde für die

Zeitschrift einen großen Gewinn an

Renommee und Prestige bedeuten.

Die Aufnahme der Notfall + Rettungsmedizin bei PubMed öffnet die Zeitschrift für noch mehr hochkarätige Beiträge und Autoren, die deren Fachwissen unseren Lesern zur Verfügung stellen. Aber: Dieses Ziel ist schwierig zu erreichen. Pubmed indexiert derzeit 643 Zeitschriften. Springer hat im Jahr 201413 Zeitschriften angemeldet, von denen nur eine einzige neu aufgenommen wurde.

Nichtsdestotrotz ist es unsere Absicht, die Notfall + Rettungsmedizin für die Lis- tung bei PubMed einzureichen. In den letzten Jahren haben Verlag und Herausgeber einige für das Vorhaben unerlässliche formale und organisatorische Voraussetzungen geschaffen. Wir denken: Die Zeit ist reif!

Es sind jedoch US-amerikanische Wissenschaftler, die die Beurteilung über die Zeitschriften treffen. Um diesem Board die Wichtigkeit der Notfall + Rettungsmedizin deutlich zu machen und damit die Finanzierung der Aufnahme in PubMed durch die Regierung zu rechtfertigen, muss die Relevanz für die amerikanischen Kollegen gegeben sein.

\section{》) Ein wichtiger Aspekt ist die Publikationssprache}

Ein wichtiger Aspekt für den Erfolg des Antrags ist die Publikationssprache. Obwohl diese in Notfall + Rettungsmedizin in der Regel deutsch ist und bleibt, sind Schriftleitung und Verlag aufgeschlossen gegenüber englischsprachigen Artikeln, insbesondere bei wissenschaftlichen Beiträgen wie Originalpublikationen. Die Arbeiten können direkt auf Englisch erstellt, über den Editorial Manager für die entsprechende Rubrik eingereicht und - nach Begutachtung so publiziert werden. Dabei sollte der Autor überlegen, ob das Thema international oder eher regional von Interesse ist.

Weiterhin ist es möglich, auch Beiträge anderer Rubriken, etwa in „Konzepte - Stellungnahmen - Leitlinien“, parallel auf Deutsch in der Printausgabe und
Englisch online zu publizieren. Der Autor kann beide Varianten bei uns einreichen. Darüber hinaus ist es erstrebenswert, dass englischsprachige Gastbeiträge - als „Blick über den Tellerrand" - internationale Themen der Notfallmedizin darstellen. Diese genannten Optionen sollen gezielt und in Maßen eingesetzt werden, damit die Notfall + Rettungsmedizin als wichtigste deutschsprachige notfallmedizinische Zeitschrift im Kern auch weiterhin so erhalten bleibt, wie Sie sie kennen und schätzen.

In der letzten Zeit haben Schriftleitung, Herausgeber und Verlag den PubMed-Antrag geplant und bereits in Angriff genommen. Wir möchten Sie, unsere Leser, darauf hinweisen, dass in den nächsten Heften einige der Leitthemenbeiträge daher in englischer Sprache erscheinen werden. Dies ist nötig, da im Komitee der NLM-NIH das gedruckte Heft beurteilt wird. Englische Beiträge verdeutlichen so die internationale Relevanz unserer Zeitschrift. Diese Leitthemenbeiträge werden natürlich für Sie auch immer in deutscher Sprache online zur Verfügung gestellt werden.

Dieser Schritt wird die Relevanz unserer Beiträge und damit unserer Zeitschrift steigern und so für Leser und Autoren eine Bereicherung darstellen. Die Schriftleitung

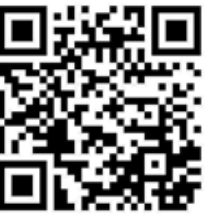

Abb. $1<$ Quick-respon$\mathrm{se}(\mathrm{QR})$-Code für Ihr Smartphone zu http:// www.editorialmanager. com/nore 
lädt Sie als Leser ein, die Notfall + Rettungsmedizin bei diesem wichtigen Vorgehen $\mathrm{zu}$ unterstützen, und wir laden alle Autoren ein, uns ihre Beiträge anzuvertrauen. Sollten Sie zu den genannten Erweiterungen Fragen haben, wenden Sie sich gerne an den Verlag oder die Schriftleitung.

Mit besten Grüßen

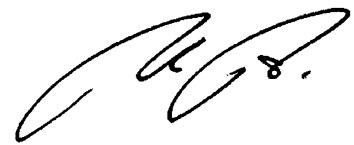

Bernd W. Böttiger

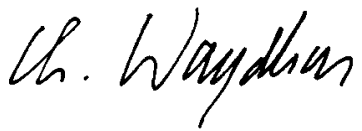

Christian Waydhas

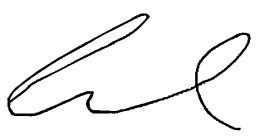

Christian Wrede

\section{Korrespondenzadresse}

Prof. Dr. B.W. Böttiger

Klinik für Anästhesiologie

und Operative Intensivmedizin

Universitätsklinikum Köln

Kerpener Straße 62

50937 Köln

bernd.boettiger@uk-koeln.de

\section{Einhaltung ethischer Richtlinien}

Interessenkonflikt. Die Autoren geben an, dass kein Interessenkonflikt besteht.

Dieser Beitrag beinhaltet keine Studien an Menschen oder Tieren.

\section{Literatur}

1. Herrmann-Lingen C, Brunner E, Hildenbrand S et al (2014) Evaluation of medical research performance - position paper of the Association of the Scientific Medical Societies in Germany (AWMF). GMS Ger Med Sci;12:Doc11. DOI: 10.3205/000196, URN: urn:nbn:de:0183-0001969
St.Pierre, M, Hofinger, G

Human Factors und Patientensicherheit in der Akutmedizin

Heidelberg: Springer Verlag 2014, 3. überarb. Auflage, 368 S., 62 Abb., (ISBN 978-3-66245879-2), 59.99 EUR

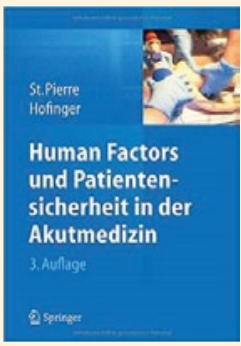

Das in den ersten beiden Auflagen unter dem Titel „Notfallmanagement" erschienene Buch ist in der 3. Auflage nochmals deutlich erweitert und komplett aktualisiert. Es zählt hiermit weiterhin zu den herausragenden Standardwerken mit allen wesentlichen Aspekten des modernen akutklinischen Risikomanagements und der Patientensicherheit.

Die neue Auflage besticht durch eine noch größere Praxisnähe zur klinischen Routinearbeit, indem viele sorgfältig erprobte Umsetzungskonzepte aufgenommen und um aktuelle Themenkomplexe erweitert wurden. Insbesondere der neu konzipierte und stark erweiterte Überblick über das The-menfeld „Organisation" spiegelt umfassend die neuesten Ergebnisse aus der Organisationspsychologie, den aktuellen Erkenntnissen des Risikomanagements und der klinischen Arbeit wider.

Das Werk fokussiert sehr gut auf die wesentlichen Mechanismen von akutmedizinischen Entscheidungsfindungen inklusive der hierin impliziten - nicht selten erfolgskritischen Human Factor Aspekte. Es beschreibt tiefgreifend und verständlich die zentralen (Klinik-) Teamaspekte mit den korrespondierenden organisatorischen und psychologischen Rahmenbedingungen.

Das Buch zeigt eindrucksvoll - in einer sehr klaren Sprache und vielen anschaulichen Darstellungen - den aktuellen Erkenntnisstand hinsichtlich des Zusammenwirkens differenter akutklinischer Systemebenen und ihren direkten oder mittelbaren Einfluss auf konkretes menschliches Handeln im klinischen Alltag. Es verfügt über eine exzellente aktuelle Literaturübersicht, ein durchdachtes Stichwortregister und viele Gliederungshilfen zum schnellen Memorieren und Wiederauffinden. Das Buch gibt allen in der Akutmedizin arbeitenden Menschen, berufs- und disziplinübergreifend, konkrete Unterstützung und ermög- licht tiefgreifendes Verständnis von aktuellen Erkenntnissen zur Patientensicherheit und den prognosebestimmenden Faktoren im klinischen Risikomanagement. Es wird für Berufsanfänger und für Erfahrene gleichermaßen wertvolle Handlungsorientierung im täglichen klinischen Alltag liefern.

Dieses Buch verdient einen festen Platz am Arbeitsplatz jeden Entscheidungsträgers in der Akutmedizin

\section{München/Hamburg \\ Prof.Dr.C.K. Lackner/Prof.Dr.Hp.Moecke/ Dr.phil. Dipl.-Psych.K.Burghofer}

